\title{
Transient resonance of rolling finned projectiles
}

\author{
N Ananthkrishnan ${ }^{1}$, S C Raisinghani² ${ }^{2}$ and S Pradeep ${ }^{3 *}$ \\ ${ }^{1}$ Department of Aerospace Engineering, Indian Institute of Technology, Bombay, India \\ ${ }^{2}$ Department of Aerospace Engineering, Indian Institute of Technology, Kanpur, India \\ ${ }^{3}$ Department of Aerospace Engineering, Indian Institute of Science, Bangalore, India
}

\begin{abstract}
Finned projectiles that are intentionally spun are known to become locked in at a resonance condition where the roll rate matches the pitch/yaw frequency. This phenomenon, called roll lock-in, results in an amplification of the trim angle, which can lead to yaw instability. A less severe phenomenon is that of transient resonance where the roll rate locks in at resonance briefly before continuing to build up to the design value. However, the trim amplification during the brief period of resonance may be sufficient to destabilize the projectile. In this paper, the problem of transient resonance for finned projectiles with a centre-of-mass asymmetry is studied. Numerical simulations are used to illustrate transient normal and reverse resonance. No yaw amplification is seen in both cases; rather the absolute value of the complex angle of attack shows a drop during passage through resonance. It is suggested that this is a general feature of the yaw response of finned projectiles undergoing transient resonance.
\end{abstract}

Keywords: resonance, projectiles, roll lock-in

\section{NOTATION}

$G=\left(\hat{r}_{\mathrm{c}} h \delta_{\mathrm{t} 0} C_{\mathrm{N} \alpha} / 2\right)\left[C_{\mathrm{lp}}+\left(I_{x} / m l^{2}\right) C_{\mathrm{D}}\right]$

$[-(1-\sigma) / M]^{0.5}$

$h=(\hat{H}-\sigma \hat{T})(1-\sigma)$

$\hat{H}=[\rho S l /(2 m)]\left[C_{\mathrm{L} \alpha}-C_{\mathrm{D}}-\left(m l^{2} / I\right)\right.$

$\left.\times\left(C_{\mathrm{Mq}}+C_{\mathrm{m} \dot{\alpha}}\right)\right][-(1-\sigma) / M]^{0.5}$

I transverse moment of inertia

$I_{x} \quad$ axial moment of inertia

$\hat{K}_{\mathrm{p}} \quad-\left[\rho S l^{3} /\left(2 I_{x}\right)\right]\left[C_{\mathrm{lp}}+\left(I_{x} / m l^{2}\right) C_{\mathrm{D}}\right][-(1-\sigma) / M]^{0.5}$

$l$ reference length, diameter

$m$ mass

$M \quad\left[\rho S l^{3} /(2 I)\right] C_{\mathrm{M} \alpha}$

$\hat{r}_{\mathrm{c}} \quad$ radial centre of mass offset, calibers

$S \quad$ reference area

$t$ time

$t_{1} \quad=[-M /(1-\sigma)]^{0.5} \int_{0}^{t}(V / l) \mathrm{d} t$

$\hat{T} \quad=[\rho S l /(2 m)]\left[C_{\mathrm{L} \alpha}+\left(m l^{2} / I_{x}\right) C_{\mathrm{mp} \alpha}\right]$

$\times[-(1-\sigma) / M]^{0.5}$

$\delta_{\mathrm{T} 0} \quad$ magnitude of non-rolling trim

$\theta \quad$ orientation angle of $\tilde{\tau}$ in missile-fixed axes

$\tilde{\theta} \quad$ orientation angle of $\tilde{\tau}$ in aeroballistic axes

$\mu \quad \tilde{\tau}\left(h / \delta_{\mathrm{T} 0}\right) \mathrm{e}^{\mathrm{i}(\theta-\tilde{\theta})}$

$\rho \quad$ air density

$\sigma \quad I_{x} / I$

$\tilde{\tau} \quad$ complex angle of attack in aeroballistic axes

The MS was received on 24 August 1998 and was accepted for publication on 5 March 1999.

* Corresponding author: Department of Aerospace Engineering, Indian Institute of Science, Bangalore 560012, India.
$\phi_{\mathrm{M}} \quad$ orientation angle of non-rolling trim

$\dot{\phi} \quad$ non-dimensional roll rate

\author{
Subscripts \\ e equilibrium value \\ s design value
}

\author{
Superscripts \\ - complex conjugate \\ - $\mathrm{d}(.) / \mathrm{d} t_{1}$
}

\section{INTRODUCTION}

Rolling finned projectiles with slight configurational asymmetry are known to experience a phenomenon called roll lock-in, where the roll rate settles down to a steady state value that matches with the natural yawing frequency of the projectile. The locked-in roll rate resonantly excites the yaw dynamics, resulting in an amplification of the nonrolling trim angle, which may be sufficient to destabilize the yawing motion of the projectile.

As the projectile is spun up to the design roll rate, which is usually much larger than the resonant value, the roll rate is expected to pass through resonance. Depending on the initial conditions at launch, the roll rate may build up to the design value or may lock in at resonance. In some cases, the phenomenon of transient resonance may occur, where the roll rate may linger for a while at the resonance 
condition before eventually building up to the design value. The amplification of the trim angle and the possibility of yaw instability during transient resonance are matters of significant research interest.

The problem of resonance lock-in of rolling finned missiles was introduced by Nicolaides [1], who attributed the phenomenon to the significant non-linear roll moments induced by the trim angle under resonance conditions. The primary cause of these induced roll moments was suggested by Price [2] to be an offset centre of mass. The problem of transient resonance in finned missiles with an offset centre of mass was studied numerically by Price [2] and Barbera [3], and in the case of an induced roll moment of aerodynamic origin by Chadwick [4]. Dynamic instabilities of missiles including roll lock-in have been discussed in a survey paper by Murphy [5].

An analytical approach to the problem of roll lock-in for a finned missile with a centre-of-mass offset was presented by Murphy [6], who showed that both normal and reverse lock-in solutions were possible. The equations of motion reported by Murphy [6] were corrected by Ananthkrishnan and Raisinghani [7], who also showed the possibility of occurrence of quasi-steady resonant lock-in solutions. A detailed review of the theory of resonant lock-in of rolling finned projectiles with illustrative calculations has been provided by Ananthkrishnan and Raisinghani [8]. A recent paper by Platus [9] discusses the problem in the context of coning instabilities in missiles and spacecraft.

In the present paper, the problem of transient resonance of rolling finned projectiles with an offset centre of mass is studied using the formulation of Murphy [6] and Ananthkrishnan and Raisinghani [7]. Using numerical simulations, the effect of passage through resonance, both normal and reverse, on the trim amplification is evaluated.

\section{EQUATIONS OF MOTION}

The equations of motion are written in an aeroballistic axis system following the notation of reference [7] as follows:

$$
\begin{aligned}
& \ddot{\phi}+\hat{K}_{\mathrm{p}}\left[\dot{\phi}-\dot{\phi}_{\mathrm{s}}-\mathrm{i} G(\mu-\bar{\mu})\right]=0 \\
& \ddot{\mu}+[\hat{H}+\mathrm{i}(2-\sigma) \dot{\phi}] \dot{\mu}+\left[(1-\sigma)\left(1-\dot{\phi}^{2}+\mathrm{i} \dot{\phi} h\right)+\mathrm{i} \ddot{\phi}\right] \mu \\
& \quad=-h(1-\sigma) \exp \left(\mathrm{i} \phi_{\mathrm{M}}\right)
\end{aligned}
$$

The roll rate $\dot{\phi}$ is scaled such that the resonance condition corresponds to $\dot{\phi}= \pm 1$. Similarly, $\mu$ is the complex angle of attack in the missile-fixed frame scaled with respect to the trim angle magnitude at resonance. In the above equations, $h$ is a measure of the trim asymmetry with orientation $\phi_{\mathrm{M}}$ and $G$ is a measure of the centre-of-mass offset.

Equilibrium solutions of this system of equations are obtained from

$$
\begin{aligned}
& \dot{\phi}_{\mathrm{e}}-\dot{\phi}_{\mathrm{s}}-\mathrm{i} G\left(\mu_{\mathrm{e}}-\bar{\mu}_{\mathrm{e}}\right)=0 \\
& \mu_{\mathrm{e}}=-h\left(1-\dot{\phi}_{\mathrm{e}}^{2}+\mathrm{i} \dot{\phi}_{\mathrm{e}} h\right)^{-1} \exp \left(\mathrm{i} \phi_{\mathrm{M}}\right)
\end{aligned}
$$

Stability of these equilibrium solutions can be determined from a linearized small-perturbation analysis, otherwise called Lyapunov's first method [10]. Stability of an equilibrium solution is then indicated by the eigenvalues of the Jacobian matrix evaluated at the equilibrium point. The elements of the Jacobian matrix (also called the stability matrix) have been listed in reference [7].

Equilibrium solutions for two combinations of the parameters $G, \phi_{\mathrm{M}}$ are given in Table 1 . The other parameters of the system are kept fixed at the following values:

$$
\begin{array}{ll}
\dot{\phi}_{\mathrm{s}}=3.0, & h=0.1, \quad \hat{H}=0.1, \\
\hat{K}_{\mathrm{p}}=0.1, & \sigma=0.1
\end{array}
$$

Case A in Table 1 shows a stable normal resonance solution and a stable design solution, while case B shows a stable reverse resonance solution and a stable design solution. The normal resonance solution in case $\mathrm{B}$ may be noted to be unstable. The two cases in Table 1 will be used to illustrate the results in the rest of this paper.

Table 1 Equilibrium solutions and their stability

\begin{tabular}{lccrll}
\hline Case & $\phi_{\mathrm{M}}$ & $G$ & \multicolumn{1}{l}{$\dot{\phi}_{\mathrm{e}}$} & $\mu_{\mathrm{e}}$ & Stability \\
\hline A & $90^{\circ}$ & 3.0 & 1.020 & $-0.8474+0.3357 \mathrm{i}$ & Stable-normal resonance \\
& & & 1.129 & $-0.1280+0.3115 \mathrm{i}$ & Unstable \\
& & & 2.920 & $-0.0005+0.0133 \mathrm{i}$ & Stable-design solution \\
B & $270^{\circ}$ & 5.0 & -0.976 & $-0.8289+0.4028 \mathrm{i}$ & Stable-reverse resonance \\
& & & -0.862 & $-0.1545+0.3889 \mathrm{i}$ & Unstable \\
& & & 0.754 & $0.0393+0.2249 \mathrm{i}$ & Unstable \\
& & & 0.990 & $0.9709+0.1952 \mathrm{i}$ & Unstable \\
& & & 3.115 & $0.0004-0.0115 \mathrm{i}$ & Stable-design solution \\
$\mathrm{C}$ & $(h=0)$ & 0 & 3.000 & 0 & Stable-design solution \\
\hline
\end{tabular}




\section{NORMAL AND REVERSE LOCK-IN}

Cases $\mathrm{A}$ and $\mathrm{B}$ in Table 1 show two stable equilibrium solutions each. Depending on the initial conditions at launch, in each case, either of the two equilibrium solutions may be attained. The effect of disturbances at launch is given naturally in terms of initial conditions on the yaw rate, with the complex angle of attack and roll rate assumed to be zero initially.

To investigate case $\mathrm{A}$ in Table 1, the magnitude of the initial yaw rate is chosen to be 1.0 , with two possible orientations: $\theta^{*}=0^{\circ}$ and $\theta^{*}=180^{\circ}$. Results of numerical simulations for these two initial conditions showing the build-up of roll rate and the magnitude of the complex angle of attack are plotted in Figs 1 and 2 respectively. It can be seen that for $\theta^{*}=180^{\circ}$, the roll rate builds up to the design value (actually 2.920 , as in Table 1 ) and the complex angle of attack dies down to a value that is nearly zero. On the other hand, for $\theta^{*}=0^{\circ}$, the phenomenon of normal resonant lock-in with the complex angle of attack building up to a value nearly equal to 1.0 can be seen.

As is well known from linear aeroballistic theory [8], the yaw dynamics is tricyclic; i.e. it consists of three modesnutation, precession and trim. Stable dynamics implies that, at steady state, both the nutation and precession modes will have damped out, and the complex angle of attack at equilibrium is precisely due to the trim component. However, in the transient phase, before attainment of steady state, the yaw dynamics is truly tricyclic, and the variation in the magnitude of the complex angle of attack $\mu$ depends

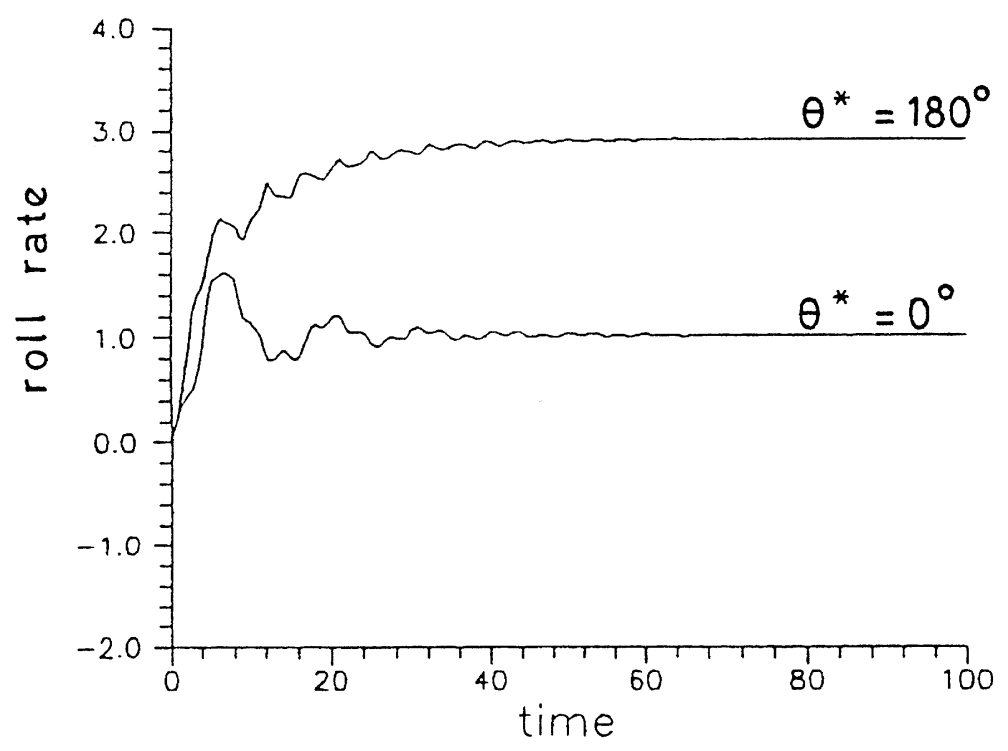

Fig. $1 \dot{\phi}$ versus $t$, case A in Table 1, with $\left|\dot{\mu}_{0}\right|=1.0$

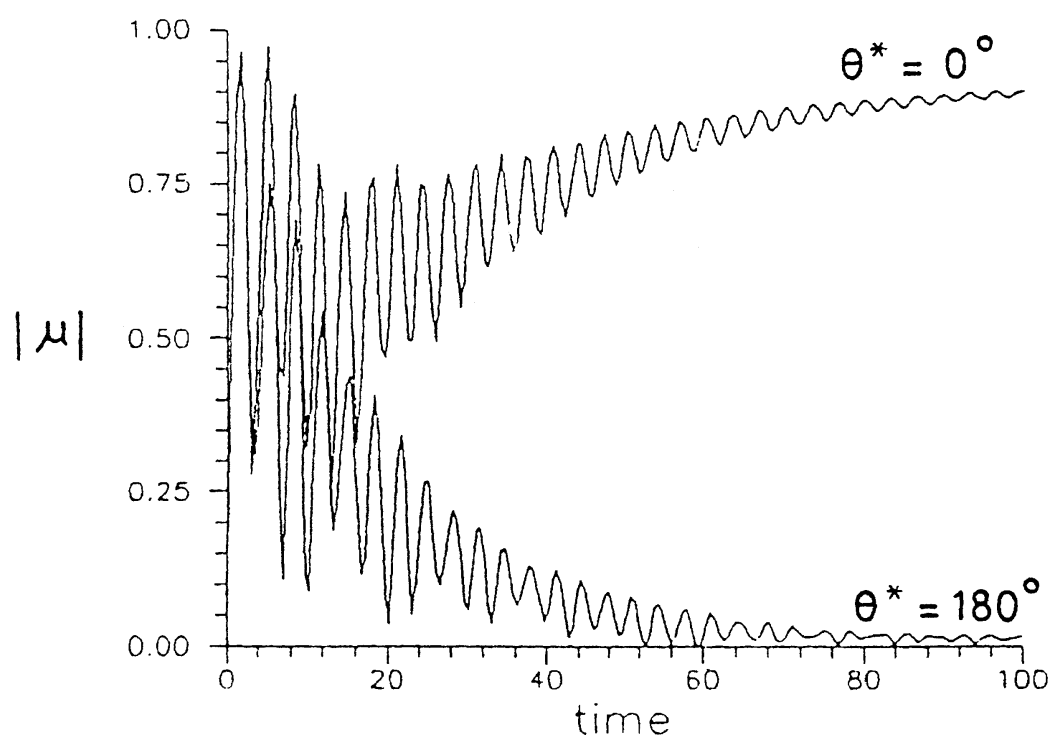

Fig. $2|\mu|$ versus $t$, case A in Table 1, with $\left|\dot{\mu}_{0}\right|=1.0$ 
on all three modes. For example, $|\mu|$ can decrease even when the trim mode is being amplified because of the greater attenuation of the nutation and precession modes. This effect can be seen in Fig. 2 where, for $\theta^{*}=0^{\circ}$, the peak of the oscillations in $|\mu|$ initially decreases before building up to the steady state value.

The dynamics for case B in Table 1 can be studied in a similar fashion. Once again the magnitude of the initial yaw rate is chosen to be 1.0 with two possible orientations: $\theta^{*}=90^{\circ}$ and $\theta^{*}=270^{\circ}$. Results of numerical simulations for these two initial conditions showing the build-up of the roll rate and the magnitude of the complex angle of attack are plotted in Figs 3 and 4 respectively. For $\theta^{*}=90^{\circ}$, Fig. 3 shows the roll rate building up to the design value, while the complex angle of attack in Fig. 4 settles down to a near- zero value. On the other hand, for $\theta^{*}=270^{\circ}$, the phenomenon of reverse resonant lock-in with the complex angle of attack building up to a value nearly equal to 1.0 can be seen. Once again, the peak of the $|\mu|$ oscillations in Fig. 4 for $\theta^{*}=270^{\circ}$ shows a dip before increasing to the equilibrium value for the same reason as before.

\section{TRANSIENT RESONANCE}

It may be expected that for some values of initial conditions the roll rate will linger at resonance for a period of time before breaking out and eventually reaching the steady state design value. It is conjectured that the projectile

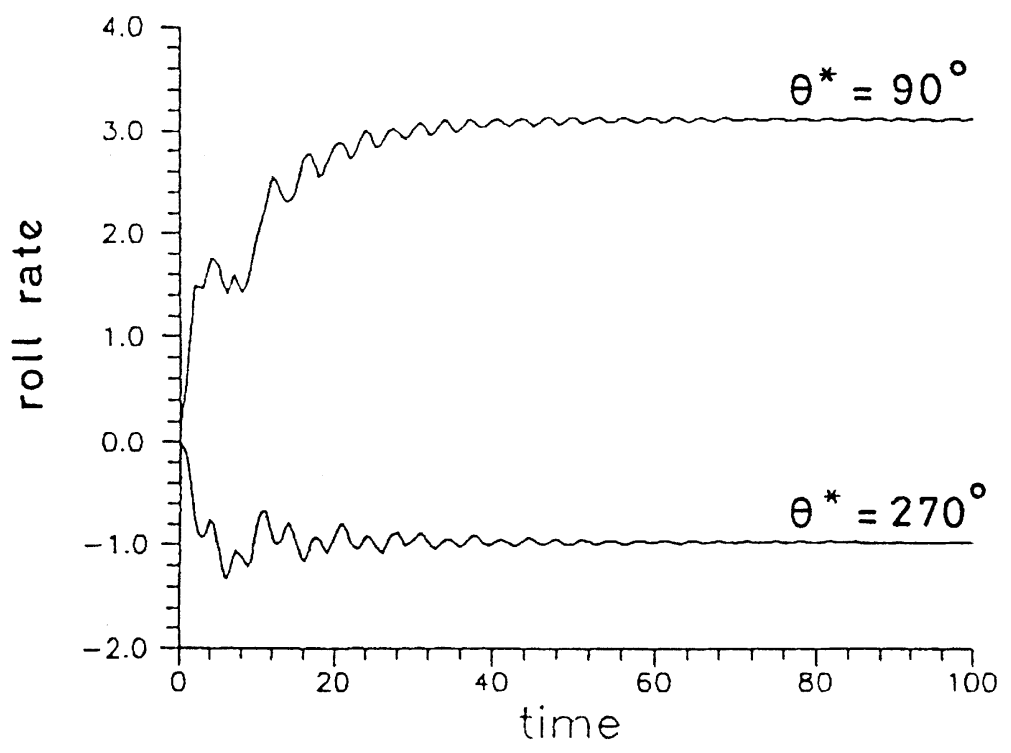

Fig. $3 \dot{\phi}$ versus $t$, case B in Table 1, with $\left|\dot{\mu}_{0}\right|=1.0$

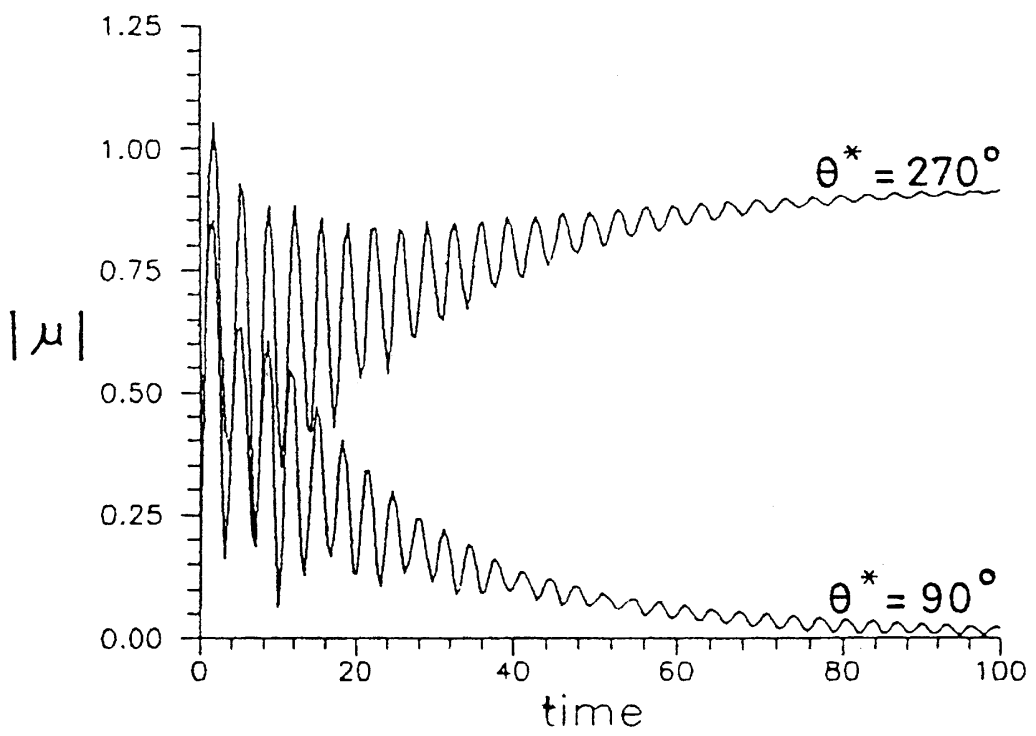

Fig. $4|\mu|$ versus $t$, case B in Table 1, with $\left|\dot{\mu}_{0}\right|=1.0$ 
would experience yawing motion of increased amplitude during the period it is at resonance, owing to amplification of the trim mode. In that case, yaw instability may arise even for initial conditions that do not lead to stable resonant lock-in solutions.

In order to evaluate the yaw response during transient resonance, the ideal projectile is introduced-one with no centre-of-mass offset $(G=0)$ and no trim asymmetry ( $h=0$ ). The only equilibrium solution with $G=0$ is the design solution listed in Table 1 under case C. With $h=0$, the yaw dynamics is independent of $\phi_{\mathrm{M}}$, and reduces to just the nutation and precession modes, which, for stable dynamics, implies a steady state value of $\mu$ equal to zero. On the other hand, for the problem of transient resonance, the steady state $\mu$ is near-zero (corresponding to the design solution), but with a yaw response that combines all three modes - nutation, precession and trim. Hence, the yaw response of the ideal projectile provides a suitable standard against which the expected yaw amplification during transient resonance, for both case $\mathrm{A}\left(\phi_{\mathrm{M}}=90^{\circ}\right)$ and case B $\left(\phi_{\mathrm{M}}=270^{\circ}\right)$, can be compared.

\subsection{Transient normal resonance}

The transient resonance phenomenon is first investigated for case $\mathrm{A}$ in Table 1. The magnitude of the initial yaw rate is chosen to be 0.6 with an orientation of $\theta^{*}=0^{\circ}$. The variation of the roll rate for this initial condition, plotted in Fig. 5, clearly shows transient normal resonance. The build-up of the roll rate for case $\mathrm{C}$ in Table 1 for identical initial conditions is also shown in Fig. 5 for comparison. The variation in the magnitude of the complex angle of attack for the two cases is depicted in Fig. 6. It can be seen that the peak values of $|\mu|$ for case A begin to fall as compared to the peaks for case $\mathrm{C}$ around the time the roll rate for case $\mathrm{A}$ is temporarily locked in at resonance. The effect of this decrease in $|\mu|$ continues to be seen for larger values of time where the mean value of $|\mu|$ for case $\mathrm{A}$ is distinctly lower than that for case $\mathrm{C}$, as they tend to the equilibrium value of $\mu \approx 0$. Thus, it can be seen that there is no yaw amplification during transient normal resonance. On the contrary, the yaw response shows a distinct drop in the magnitude of the complex angle of attack during and after passage through resonance. No yaw amplification was noticed for transient normal resonance resulting from other initial yaw rates either.

\subsection{Transient reverse resonance}

Transient resonance for case B in Table 1 is investigated next. Initial conditions for the yaw rate are chosen with a magnitude of 0.8 and an orientation $\theta^{*}=270^{\circ}$. The buildup of the roll rate with these initial conditions for cases $\mathrm{B}$ and $\mathrm{C}$ is plotted in Fig. 7. The roll response for case B shows briefly the transient reverse resonance phenomenon. The variation in the magnitude of the complex angle of attack for the two cases is depicted in Fig. 8. The fall in the peak values of $|\mu|$ during the period of reverse resonance is dramatic. However, once the roll rate for case B catches up with that for case $\mathrm{C}$, the oscillations in $|\mu|$ for the two cases are similar, but for the lower amplitudes in case B. Once again, there is no yaw amplification during transient reverse resonance. Instead, there is a sharp fall in the magnitude of the complex angle of attack during passage near reverse resonance. Transient reverse resonance, like reverse lock-in [7], was found to occur for a very restricted set of initial yaw rates.

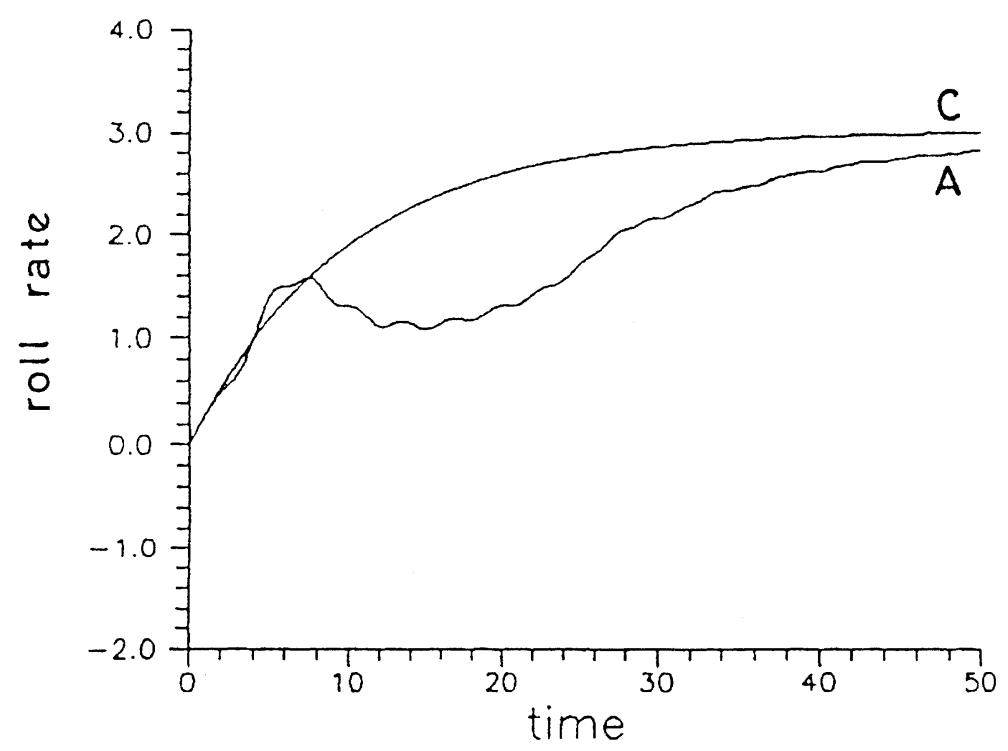

Fig. $5 \dot{\phi}$ versus $t$, cases A and C in Table 1, with $\left|\dot{\mu}_{0}\right|=0.6$ 


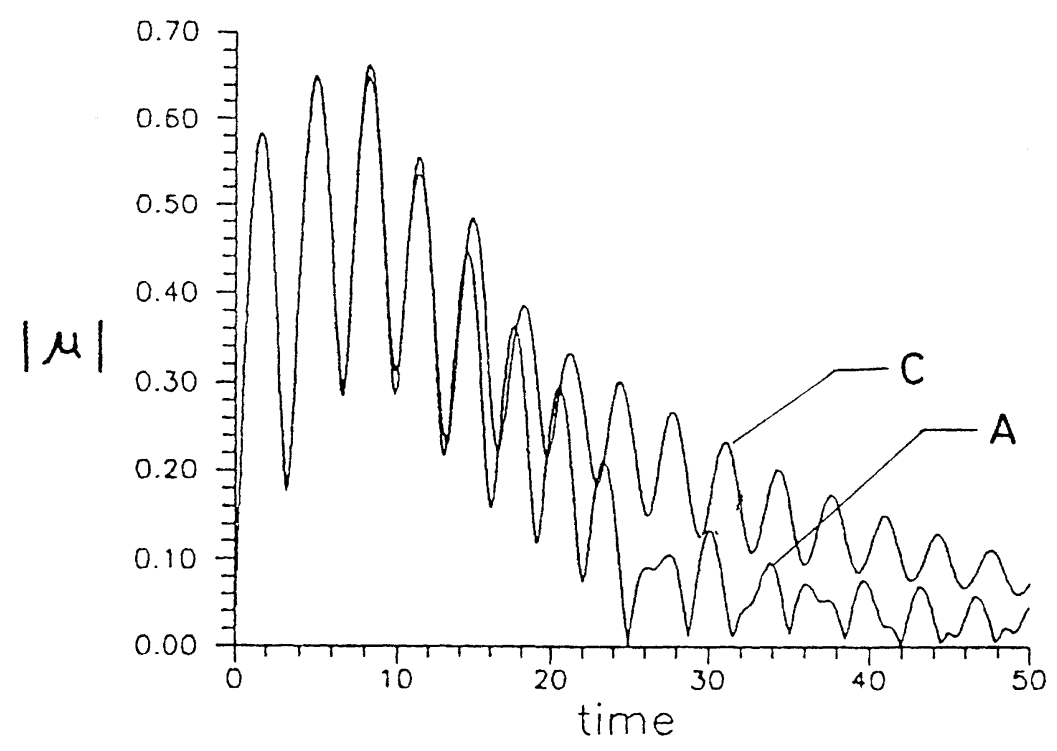

Fig. $6|\mu|$ versus $t$, cases A and C in Table 1, with $\left|\dot{\mu}_{0}\right|=0.6$

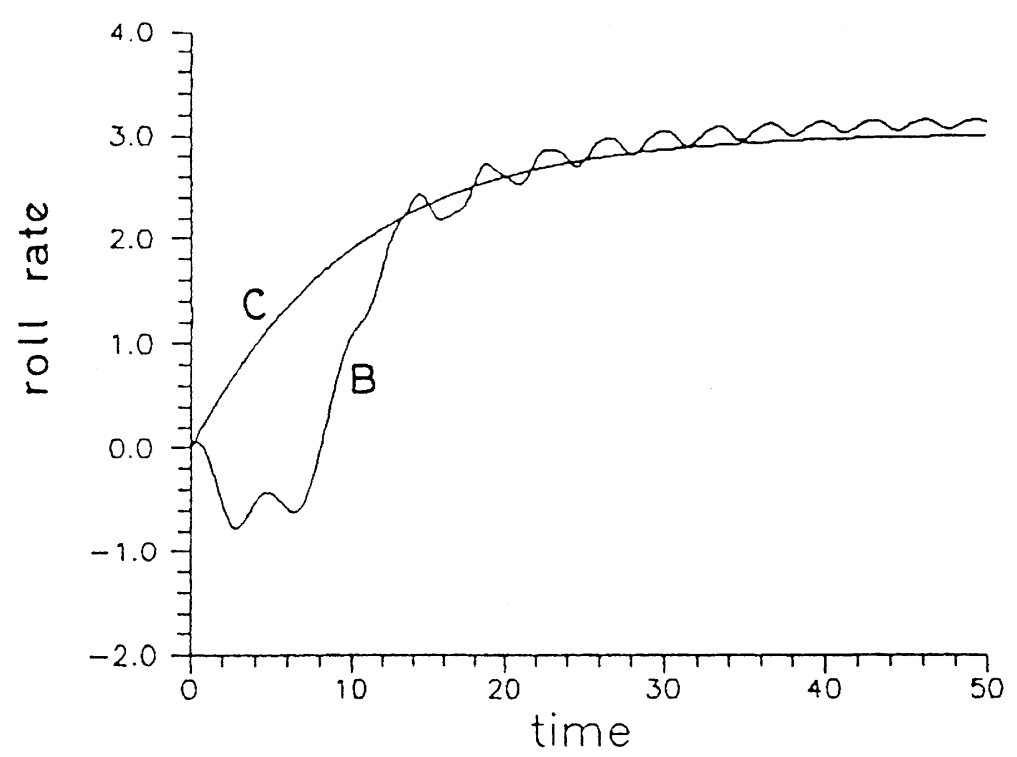

Fig. $7 \dot{\phi}$ versus $t$, cases B and C in Table 1, with $\left|\dot{\mu}_{0}\right|=0.8$

\section{DISCUSSION}

The lack of yaw amplification during transient resonance can be explained by the same effect that was noticed in Figs 2 and 4, which resulted in a decrease in the peak values of $|\mu|$ before the build-up to the steady state value. In other words, any amplification of the trim can be more than compensated for by a suitable variation of the magnitude and orientation of the nutation and precession modes.

It is tempting to explain the yaw behaviour during transient resonance in terms of the increased damping of the nutation and precession modes in the vicinity of the resonant lock-in solutions, as can be seen by evaluating the eigenvalues of the Jacobian matrix for the various stable solutions listed in Table 1. However, this argument is fallacious, since it must be noted that the dynamics represented by the eigenvalues is accurate only for small perturbations from an equilibrium state. The state variables during transient resonance are not expected to be in a small neighbourhood of the stable resonance solution, lest they find themselves within the basin of attraction of the equilibrium at resonance and end up at a stable lock-in solution at steady state.

Instead, it is instructive to consider the roll equation rewritten as follows: 


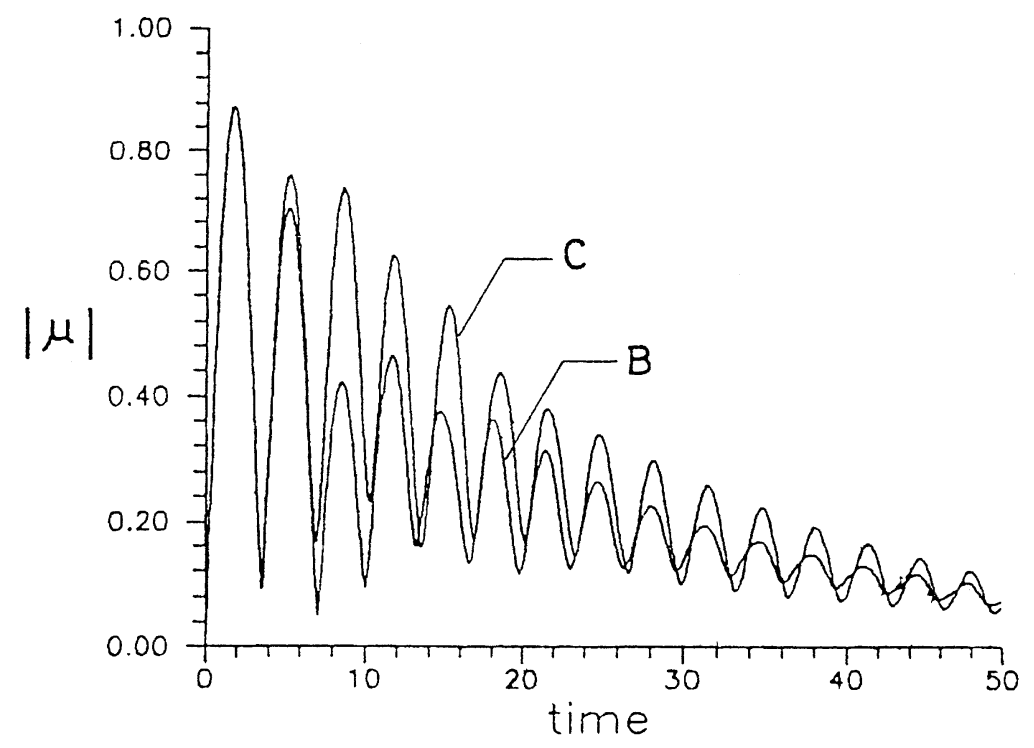

Fig. $8|\mu|$ versus $t$, cases B and C in Table 1, with $\left|\dot{\mu}_{0}\right|=0.8$

$$
\ddot{\phi}=\hat{K}_{\mathrm{p}}\left[\left(\dot{\phi}_{\mathrm{s}}-\dot{\phi}\right)-2 G \operatorname{Im}(\mu)\right]
$$

At normal lock-in, $\dot{\phi} \approx 1$ with $\ddot{\phi}=0$, which gives $\operatorname{Im}(\mu) \approx 1 / G$. At reverse lock-in, $\dot{\phi} \approx-1$ with $\ddot{\phi}=0$, which gives $\operatorname{Im}(\mu) \approx 2 / G$. These can be verified for $G=3.0$ for case A and $G=5.0$ for case B in Table 1 . During transient normal resonance, $\dot{\phi} \approx 1$, and the first term on the right-hand side, $\dot{\phi}_{\mathrm{s}}-\dot{\phi} \approx 2$, is nearly constant. However, now $\ddot{\phi} \neq 0$, and as $\dot{\phi}$ stops building up for a brief while around the resonance value, the positive $\ddot{\phi}$ must decrease. It follows from the second term on the right-hand side that for large $\ddot{\phi},|\mu|$ must begin to decrease. This argument can also be applied for the problem of transient reverse resonance with similar conclusions. Thus, the above discussion suggests that the lack of yaw amplification during transient resonance is a general phenomenon and is not limited to the initial conditions investigated in this study. However, confirmation of this point can only be sought from detailed numerical studies for projectiles with different parameter values and different initial conditions.

It must also be mentioned that the pitch/yaw equation considered for the present study did not include the nonlinear induced side moments suggested by Nicolaides [1]. The induced side moments effectively decrease the damping in yaw that could result in an unlimited build-up of yaw under resonance conditions and lead to a phenomenon called catastrophic yaw. Extension of the model in references [6] and [7] to incorporate these non-linear side moment terms is yet to be done.

\section{CONCLUSIONS}

Transient resonance of finned projectiles with a centre-ofmass offset has been studied. Numerical simulations are carried out that capture the phenomenon of transient resonance, both normal and reverse. It is seen that there is no yaw amplification during passage through resonance. Rather, a decrease in the magnitude of the complex angle of attack $|\mu|$ is noticed for both transient normal and reverse resonance. It is suggested that a decrease in $|\mu|$ is a general feature of the yaw response of finned projectiles during passage through resonance.

\section{REFERENCES}

1 Nicolaides, J. D. Two nonlinear problems in the flight dynamics of modern ballistic missiles. Report 59-17, Institute of the Aeronautical Sciences, January 1959.

2 Price, D. A. Sources, mechanisms, and control of roll resonance phenomena for sounding rockets. J. Spacecraft and Rockets, 1967, 4(11), 1516-1525.

3 Barbera, F. J. An analytical technique for studying the anomalous roll behavior of re-entry vehicles. J. Spacecraft and Rockets, 1969, 6(11), 1279-1284.

4 Chadwick, W. R. Flight dynamics of a bomb with cruciform tail. J. Spacecraft and Rockets, 1967, 4(6), 768-773.

5 Murphy, C. H. Symmetric missile dynamic instabilities. $J$. Guidance, Control, and Dynamics, 1981, 4(5), 464-471.

6 Murphy, C. H. Some special cases of spin-yaw lock-in. $J$. Guidance, Control, and Dynamics, 1989, 12(6), 771-776.

7 Ananthkrishnan, N. and Raisinghani, S. C. Steady and quasisteady resonant lock-in of finned projectiles. J. Spacecraft and Rockets, 1992, 29(5), 692-696.

8 Ananthkrishnan, N. and Raisinghani, S. C. Theory of resonant lock-in of rolling finned projectiles. J. Instn Engrs (India), 1994, 74(2), 37-43.

9 Platus, D. H. Missile and spacecraft coning instabilities. $J$. Guidance, Control, and Dynamics, 1994, 17(5), 1012-1018.

10 Mohler, R. R. Nonlinear Systems, Vol. I, Dynamics and Control, 1991 (Prentice-Hall, Englewood Cliffs, New Jersey). 